\title{
Aberrantly expressed Wnt5a in nurse-like cells drives resistance to Venetoclax in chronic lymphocytic leukemia
}

Yao Guo ${ }^{1,5}$, Hanzhong Pei ${ }^{1,5}$, Bo Lu (iD ${ }^{2,5}$, Dengyang Zhang ${ }^{1}$, Yuming Zhao ${ }^{1}$, Fuqun $\mathrm{Wu}^{3}$, Honghua Sun ${ }^{3}$, Junbin Huang ${ }^{4}$, Peng Li ${ }^{1}{ }^{1}$, Chenju $\mathrm{Yi}^{1}$, Chengming Zhu (D) ${ }^{1}$, Yihang Pan ${ }^{1}$, Shunjie Wu${ }^{2}$, Chun Chen $\mathbb{D}^{4 凶}$, Xiaojun Xu (iD) ${ }^{2 凶}$ and Yun Chen (D) ${ }^{1 凶}$

(c) The Author(s) 2022

Chronic lymphocytic leukemia (CLL) is characterized by the accumulation of neoplastic B lymphocytes with high levels of Wnt5a in the plasma. Currently, the cell source of Wnt5a remains controversial. The receptor of Wnt5a is ROR1, whose expression is associated with disease progression and resistance to venetoclax, a BCL-2 inhibitor approved for the treatment of CLL. In this study, we found that the levels of Wnt5a in the plasma of CLL patients were positively correlated with absolute monocyte counts, but not lymphocyte counts. We cultured monocyte-derived nurse-like cells (NLCs) from patients with CLL, and detected Wnt5a expressed in NLCs. Flow cytometry and transwell assays showed that the antibody neutralizing Wnt5a inhibited the enhanced survival and migration in CLL cells co-cultured with NLCs. Furthermore, we performed a drug screening with CLL cells cultured with or without NLCs with a library containing 133 FDA-approved oncology drugs by using high-throughput flow cytometry. We observed a significant resistance to venetoclax in CLL cells co-cultured with NLCs. Immunoblot revealed the activation of NF-KB with enhanced expression of MCL-1 and BCL-XL in CLL cells co-cultured with NLCs. Neutralizing Wnt5a or blocking NF-KB pathway significantly decreased the expression of MCL-1 and BCL-XL, which leads to enhanced sensitivity to venetoclax in CLL cells co-cultured with NLCs. In conclusion, our data showed that NLCs could be one of the sources of Wnt5a detected in patients with CLL, and Wnt5ainduced NF-KB activation in the CLL microenvironment results in resistance to venetoclax in CLL cells.

Cell Death Discovery (2022)8:82; https://doi.org/10.1038/s41420-022-00884-y

\section{INTRODUCTION}

Chronic lymphocytic leukemia (CLL) is a malignancy of B lymphocytes that is characterized by the accumulation of small, mature-appearing neoplastic lymphocytes with CD5 expression in the blood, bone marrow, and secondary lymphoid tissues [1]. It is the most common leukemia in the Western world with a median age of diagnosis around 70 . In the United States, there are approximately 20,000 new cases and 4000 deaths from CLL per year [2]. Despite the significant progress in chemo-immunotherapy and inhibitors targeting Bruton's tyrosine kinase (BTK) made over the past decade [3-5], some patients with high-risk features still experience refractory or progressive disease due to the development of drug resistance. Novel strategies overcoming resistant mechanisms are urgently needed.

Venetoclax is a potent small molecule inhibitor targeting $B C L-$ 2, an anti-apoptotic protein highly expressed in CLL cells [6]. Treatment with venetoclax has been shown to be effective with limited toxicity in patients with CLL with $72 \%$ overall response and $19.4 \%$ complete remission in real-world setting [7]. However, although remissions are durable, patients treated with venetoclax always experience relapses eventually. Previous studies found that venetoclax is highly potent against unstimulated CLL cells in vitro. However, leukemic cells activated with signals mimicking microenvironment stimuli are less sensitive to the drug $[8,9]$. These studies indicate a critical role of CLL microenvironment in the resistance to venetoclax, but the detailed mechanisms are not fully uncovered.

In the bone marrow and secondary lymphoid tissues, CLL cells engage in complex cellular and molecular interactions with nonneoplastic accessory cells in the microenvironment [10-12]. These interactions provide CLL cells survival, proliferation, and drug resistance. Nurse-like cells (NLCs) are key components in the microenvironment that have well-documented protective effects to CLL cells [13-15]. They have been shown to attract CLL cells by the release of chemokines, such as CXCL12 and CXCL13 [16]. They further secrete proteins, such as B-cell activating factor (BAFF) and a proliferation-inducing ligand (APRIL), which enhance CLL cell survival [17]. However, inhibiting these factors cannot abolish the supportive effects of NLCs completely [18], suggesting the involvement of other factors.

Receptor tyrosine kinase-like orphan receptor 1 (ROR1) is an oncoembryonic antigen expressed on CLL cells, but not on most normal postpartum tissues [19]. The ligand that binds to ROR1 is Wnt5a, which we find present at high levels in the plasma of patients with CLL relative to that of age-matched healthy adults

\footnotetext{
${ }^{1}$ Edmond H. Fischer Translational Medical Research Laboratory, Scientific Research Center, The Seventh Affiliated Hospital, Sun Yat-sen University, Shenzhen 518107 Guangdong, China. ${ }^{2}$ Department of Hematology, The Seventh Affiliated Hospital, Sun Yat-sen University, Shenzhen 518107 Guangdong, China. ${ }^{3}$ Clinical laboratory, The Seventh Affiliated

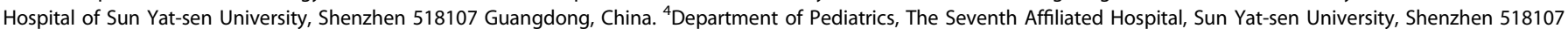
Guangdong, China. ${ }^{5}$ These authors contributed equally: Yao Guo, Hanzhong Pei, Bo Lu. ${ }^{凶}$ email: chenchun@mail.sysu.edu.cn; xuxj29@mail.sysu.edu.cn; cheny653@mail.sysu.edu.cn
}

Received: 5 January 2022 Revised: 28 January 2022 Accepted: 10 February 2022

Published online: 24 February 2022 
A

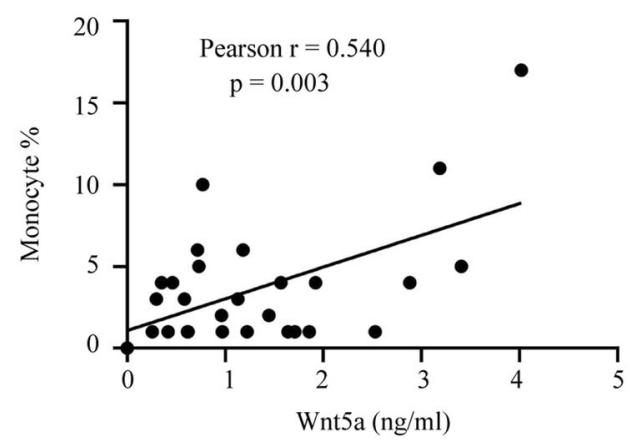

$\mathrm{C}$

CLL1

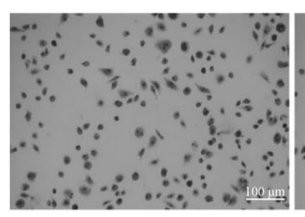

$10 \%$ monocyte
CLL2

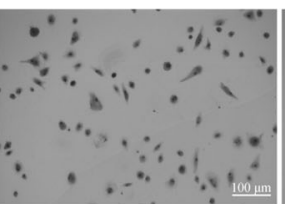

$5 \%$ monocyte
$\mathrm{B}$

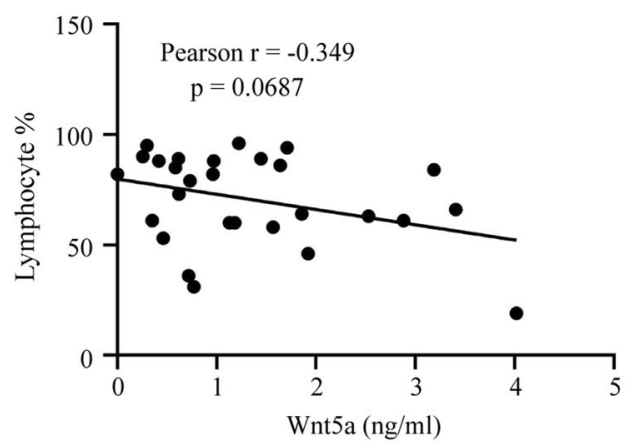

Fig. 1 Monocyte-derived NLCs correlate with Wnt5a levels in plasma from CLL patients. A, B The concentration of Wnt5a in plasma of patients positively correlates with their monocyte percentage in whole white blood cells $(\mathbf{A} n=28$, Pearson correlation $=0.540, p=0.003)$, but not their lymphocyte percentage (B $n=28$, Pearson correlation $=-0.349, p=0.0687)$. C Wright-Giemsa staining of NLCs cultured from PBMCs of patients with CLL with different monocyte percentage. Dot figures represent numbers of NLCs in the field (40X) of each group. Error bars denote standard deviation $(n=3)$.

$[20,21]$. Wnt5a can induce ROR1-dependent cell signals, thereby enhancing leukemia-cell proliferation, migration, and survival [21-27]. Previous studies showed autocrine of Wnt5a in neoplastic B cells [28], whereas WNT5A transcripts only present in neoplastic B cells in around $38 \%$ of patients with CLL [29], and high-levels of Wnt5a could be detected universally in their plasma $[20,21]$, suggesting cell-sources other than CLL neoplastic cells. We previously found that NLCs could release this non-canonical Wnt factor [13], but whether NLCs serve as the main source of Wnt5a in plasma of patients with CLL remains unknown, and the function of NLCs-released Wnt5a in the mechanisms of drug resistance still needs to be investigated. In the present study, we examined the expression of Wnt5a in NLCs in comparison with neoplastic CLL cells in patients and explored the involvement of Wnt5a in NLCsinduced survival, migration, and venetoclax-resistance in CLL cells.

\section{RESULTS}

Monocyte-derived NLCs correlate with Wnt5a levels in plasma from CLL patients

NLCs are derived from circulating monocytes from blood of patients with CLL [15]. We analyzed whole blood counts and levels of Wnt5a in plasmas of 28 CLL patients by ELISA. The result showed that levels of Wnt5a had a positive correlation with monocyte percentage, but not lymphocyte percentage (Fig. 1A, B), suggesting that Wnt5a was from monocytes or monocyte-derived cells instead of neoplastic B cells in patients with CLL. We cultured NLCs using PBMCs from patients with different monocyte counts and found that PBMCs with high monocyte counts generated high number of NLCs (Fig. 1C). We hypothesized that high monocyte counts could result in high number of NLCs, which eventually led to high levels of Wnt5a in plasma of patients with CLL. These data indicate that monocyte-derived NLCs could be the main source of high levels of Wnt5a in patients with CLL.

NLCs express high-levels of Wnt5a compared with neoplastic leukemia cells

For this study, we generated NLCs from blood mononuclear cells of CLL patients using established methods [14]. NLCs were isolated free of leukemia cells via adherence. Ultra-high resolution confocal fluorescent microscopy of NLCs showed that Wnt5a was expressed in NLCs (Fig. 2A). In addition, we measured Wnt5a levels in cell lysates from purified CLL cells or NLCs of the same patient with immunoblot. The result showed that Wnt5a was highly expressed in NLCs but neglectable in CLL cells (Fig. 2B).

\section{Wnt5a is involved in NLCs-induced survival and migration of CLL cells}

NLCs can attract CLL cells and protect them from apoptosis by elaborating factors including CXCL12, CXCL13, BAFF, and APRIL [1]. We hypothesized that Wnt5a expressed by NLCs could also be involved in these effects. We used trypan blue staining to assess the viability of CLL cells cultured with or without NLCs, and with or without the antibody neutralizing Wnt5a. We found that antiWnt5a, but not a mAb of irrelevant specificity, could significantly inhibit anti-apoptotic effects of CLL cells when co-cultured with NLCs (Fig. 3A). Next, we performed trans-well assays to assess migration of CLL cells when cultured with or without NLCs, and with or without a neutralizing mAb specific for Wnt5a. As the result, anti-Wnt5a could significantly inhibit migration of CLL cells enhanced by co-culture with NLCs (Fig. 3B). We also examined phosphorylation of ERK, HS1, and p65 in CLL cells co-cultured with NLCs, which account for Wnt5a-induced survival and migration of CLL cells according to our previous studies $[13,23,30]$. We found 
A

Merge
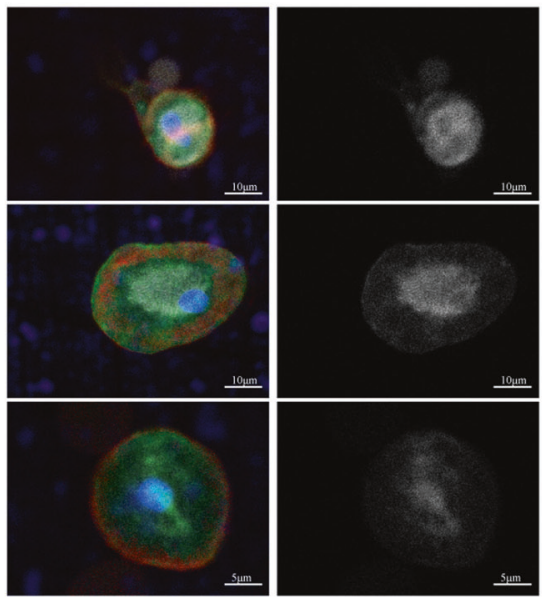

DAPI

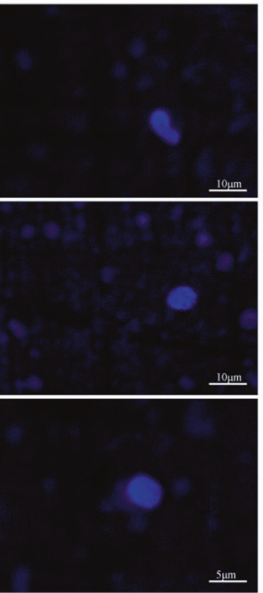

Wnt5a
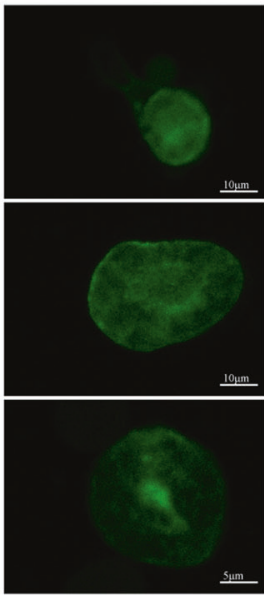

CD163

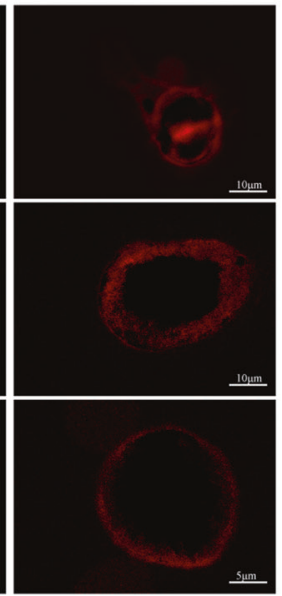

$\mathrm{B}$

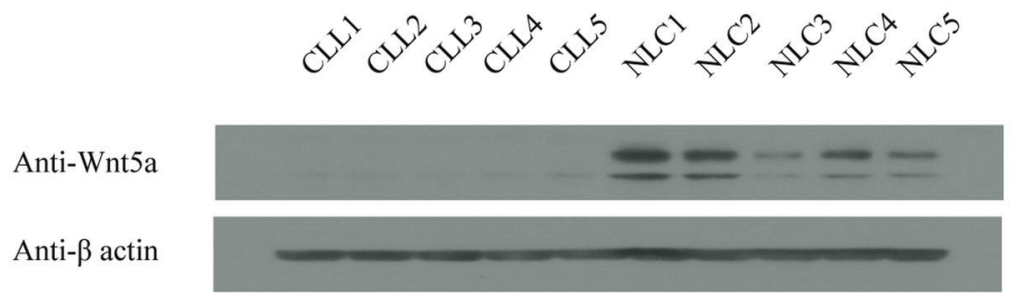

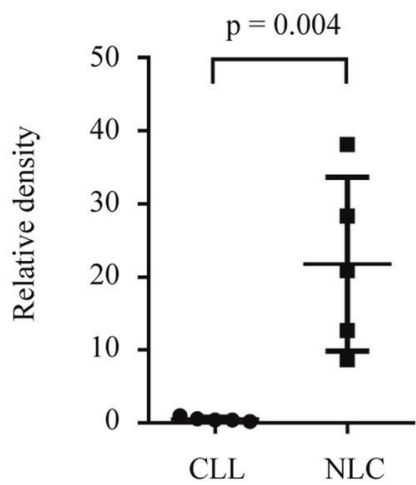

Fig. 2 NLCs but not neoplastic leukemia cells express Wnt5a. A Fluorescent microscopy of Wnt5a expressed by NLCs. CD163 and CD68 were used as markers for NLCs. B Immunoblot analysis of Wnt5a in paired NLCs and CLL cells. Dot figures represent ratios of densities (Wnt5a/ $\beta$-actin). Error bars denote standard deviation $(n=5)$.

that CLL cells co-cultured with NLCs, but not CLL cells cultured alone, had high-level of phosphorylated ERK, HS1, and p65. The phosphorylation of ERK, HS1, and p65 in co-cultured CLL cells and cell migration could be blocked by neutralizing anti-Wnt5a, but not by control mAb of irrelevant specificity (Fig. 3C).

\section{NLCs lead to drug resistance of CLL cells in a high-throughput screening}

We used high-throughput flow cytometry to evaluate the drugsensitivity of primary CLL cells cultured with or without NLCs in three patients with CLL (Fig. 4A, B). A library composed of 133 FDA-approved oncology drugs was used for the high-throughput screening. We found that romidepsin, carfilzomib, omacetaxine mepesuccinate, dasatinib, venetoclax, and dactinomycin potently inhibited CLL cells (Fig. 4C). Co-culture of CLL cells with NLCs decreased the potency of these drugs, especially carfilzomib, omacetaxine mepesuccinate, dactinomycin, and venetoclax (Fig. 4D). Note that only venetoclax among these drugs is approved for the treatment of CLL by FDA.

\section{Wnt5a upregulated MCL1 and BCL-XL in CLL cells co-cultured with NLCs}

We used flow cytometry to analyze apoptosis of CLL cells induced by venetoclax with or without NLCs and mAb neutralizing Wnt5a.
We found that NLCs significantly protected CLL cells from apoptosis, which is consistent with our high-throughput drug sensitivity screening (Fig. 5A, B). Furthermore, mAb neutralizing Wnt5a inhibited the protective effect of NLCs in CLL cells treated by venetoclax (Fig. 5A, B). Immunoblot showing that CLL cells cocultured with NLCs have significant upregulation of MCL1 and BCL-XL (Fig. 5C, D), which are BCL2 family members involved in drug resistance of venetoclax. These effects were blocked by $\mathrm{mAb}$ neutralizing $\mathrm{Wnt5a}$, indicating an important role of Wnt5a in NLCs-induced protective effects against venetoclax in CLL cells.

\section{Wnt5a induced resistance to venetoclax through activation of} NF-KB in CLL cells

We previously reported that Wnt5a could activate NF-KB pathway in CLL cells [13], which might serve as a mechanism to upregulate MCL1 and BCL-XL [31, 32]. We found that inhibition of NF-KB pathway by small molecule inhibitors significantly downregulated the expression of MCL1 and BCL-XL in CLL cells co-cultured with NLCs by immunoblot (Fig. $6 \mathrm{~A}$ ). In addition, treatment with NF-KB inhibitor significantly blocked the protective effect of NLCs against venetoclax in CLL cells by flow cytometry (Fig. 6B). Note that NF-KB inhibitor BMS-345541 alone did not change the cell viability of CLL cells. 
A

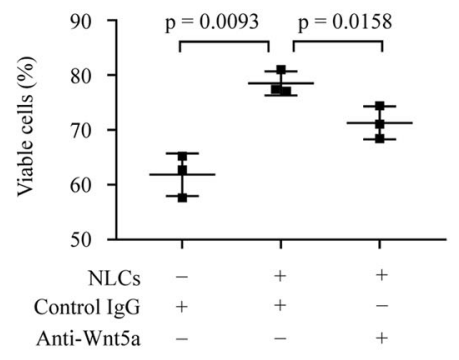

$\mathrm{C}$

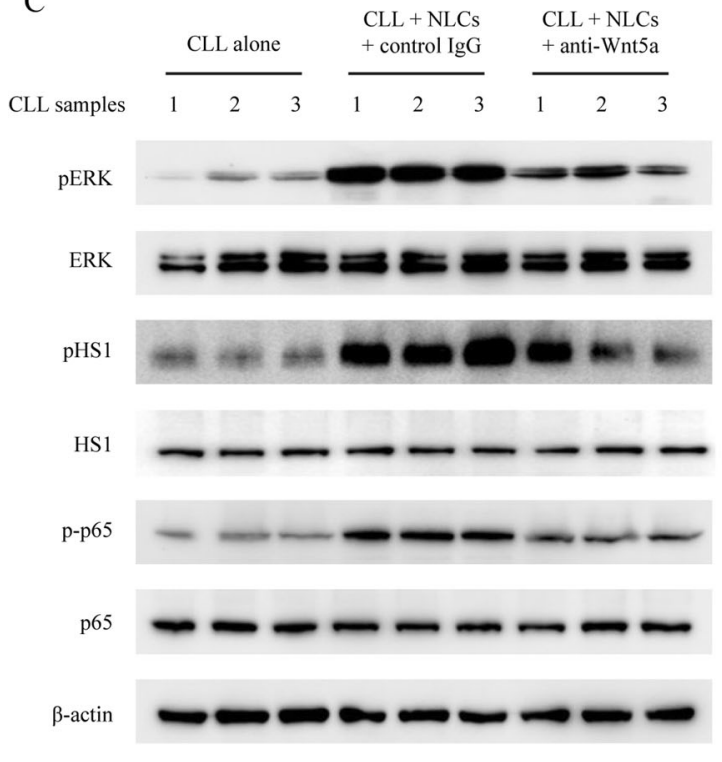

B

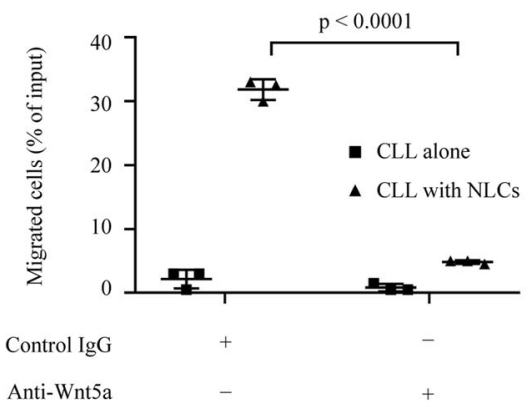

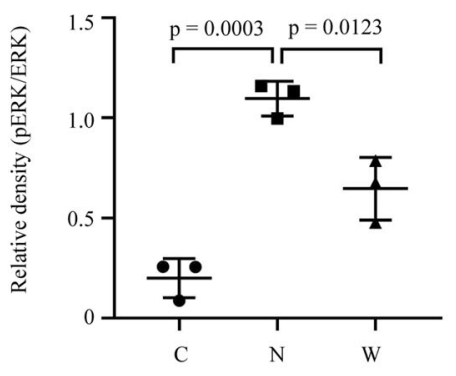
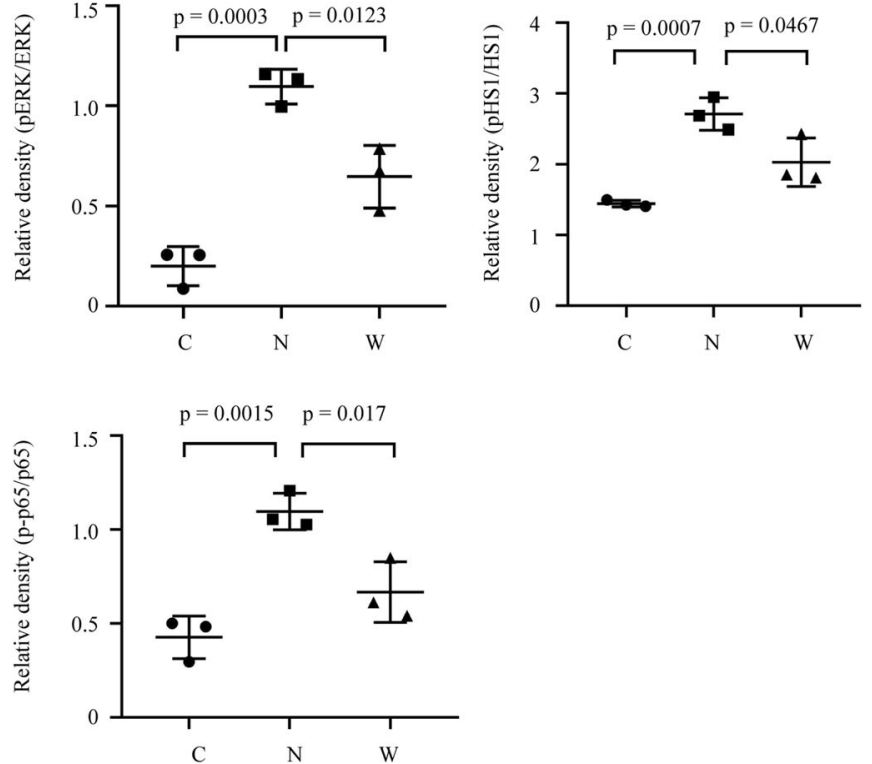

Fig. 3 Wnt5a is involved in NLCs-induced survival and migration of CLL cells. A Cell viability analysis by trypan blue staining of CLL cells with or without NLCs treated by anti-Wnt5a or control mAb of irrelevant specificity for $24 \mathrm{~h}(n=3)$. B Percentage of CLL cells migrating toward NLCs. CLL cells were treated by anti-Wnt5a or control mAb of irrelevant specificity for $4 \mathrm{~h}$. Error bars denote standard deviation ( $n=3$ ). C Immunoblot analysis of phospho-ERK, total ERK, phospho-HS1, total HS1, phospho-p65, and total p65 in lysates of CLL cells with or without NLCs treated by anti-Wnt5a or control mAb of irrelevant specificity for $24 \mathrm{~h}$. Dot figures represent ratios of densities (pERK/ERK, pHS1/HS1, and p-p65/p65).

\section{DISCUSSION}

In the present study, we found that aberrantly expressed Wnt5a in NLCs leads to resistance to venetoclax in CLL through upregulation of MCL1 and BCL-XL via NF-KB activation. First, we found that aberrantly high levels of Wnt5a in CLL patients were correlated with monocyte counts, but not lymphocyte counts, suggesting monocyte-derived cells as main sources of Wnt5a. In addition, we identified that monocyte-derived NLCs express Wnt5a. Neutralizing Wnt5a significantly inhibited NLCs-induced survival, migration, and corresponding cell signals in CLL cells. Furthermore, with a high-throughput drug screening assay, we identified significant drug resistance to venetoclax in CLL cells co-cultured with NLCs. Finally, we found that Wnt5a-induced upregulation of MCL1 and $\mathrm{BCl}-\mathrm{XL}$ by activation of $\mathrm{NF}-\mathrm{KB}$ is involved in resistance to venetoclax in CLL cells co-cultured with NLCs (Fig. 7).

Wnt5a activates non-canonical WNT signaling pathways, through binding to different members of the Frizzled- and RORfamily receptors [33]. Studies have shown that Wnt5a signaling is emerging as an important event in malignancies, including cancer cell proliferation, invasion, metastasis, metabolism, inflammation, and chemo-resistance [34]. Also, Wnt5a signaling is reported to modulate the interaction between cancer cells and accessory cells in the microenvironment, contributing to disease progression in solid tumors. In gastric carcinoma, microenvironmental lymphocytes secrete Wnt5a to stimulate the proliferation of malignant cells by activating RhoA [35]. In colon cancer, enhanced stromal Wnt5a expression promotes directional migration and invasion of cancer cells [36]. In this study, we identified that Wnt5a was involved in the crosstalk between NLCs and CLL cells. Blocking Wnt5a signaling inhibited NLCs-induced CLL proliferation, migration, and drug resistance, suggesting Wnt5a signaling as a potential therapeutic target in the microenvironment of CLL cells.

The emergence of targeted therapy for patients with CLL has altered the therapeutic landscape of the disease, but the drug resistance remains a challenge for recurrent and relapsed CLL $[5,37,38]$. Our ex vivo high-throughput drug screening found a drug-resistance to venetoclax in CLL cells induced by NLCs. Venetoclax is a selective BCL-2 inhibitor that has been approved for the treatment of first-line and relapsed/refractory CLL [6]. Monotherapy with venetoclax facilitates a rapid reduction in the disease burden with a high overall response of about $80 \%$ and a complete response of $6-20 \%$ in patients with relapsed or refractory CLL, including those with poor prognosis [39]. However, the resistance to venetoclax can be found in some CLL patients with mechanisms not fully uncovered [38]. Our study observed NLCs-induced CLL resistance to venetoclax, which was inhibited 
A

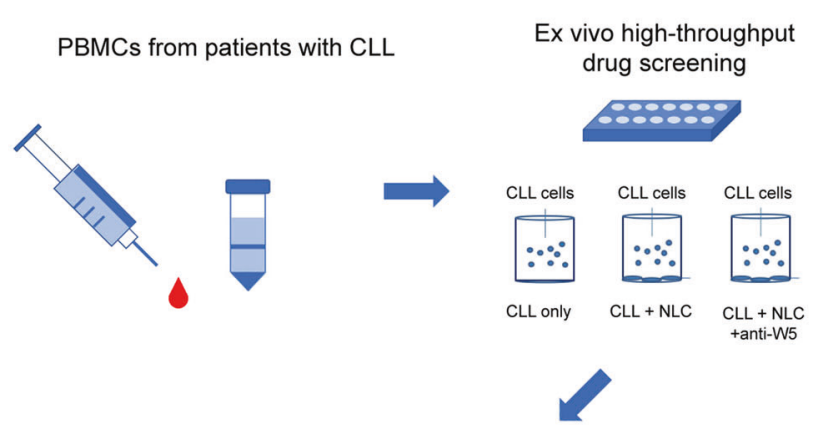

High-throughput flow cytometer

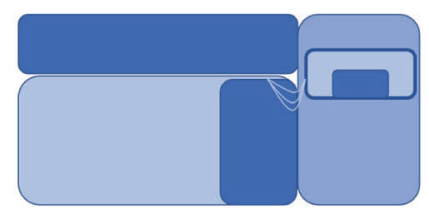

B

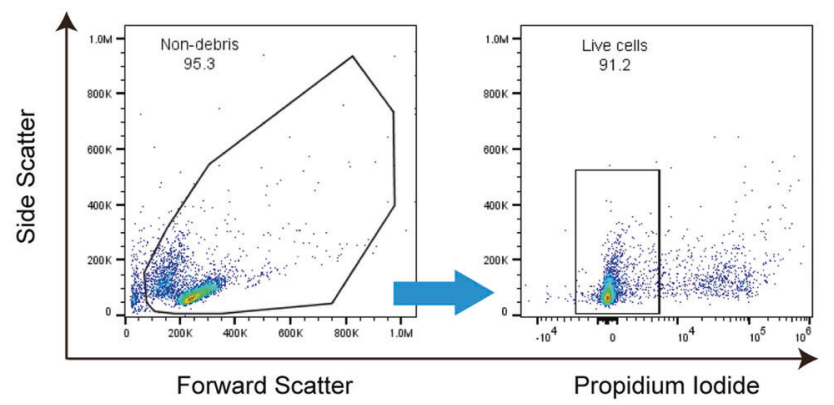

D
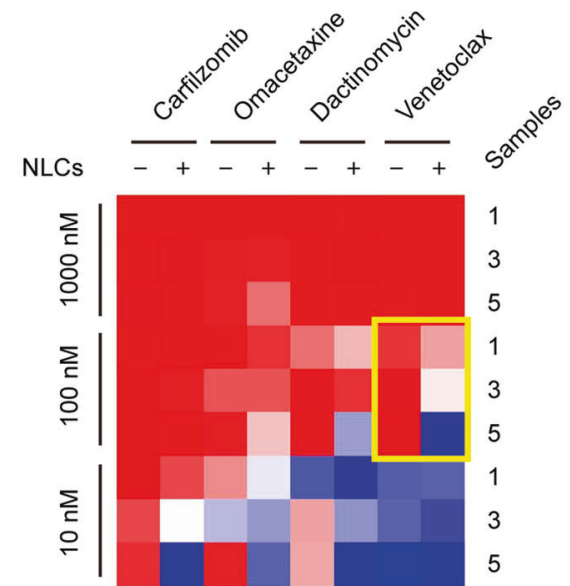

C

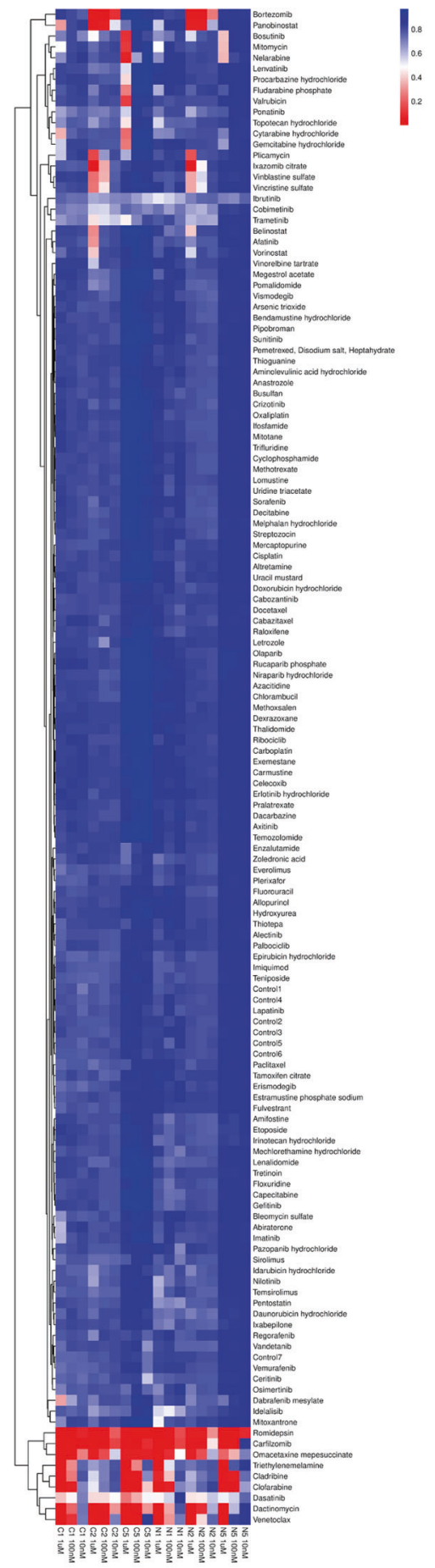

Fig. 4 Wnt5a is involved in NLCs-induced drug-resistance of CLL cells. A The flow chart of the high-throughput drug sensitivity analysis. B The gating strategy of live cells in flow cytometry. C The heatmap of the high-throughput drug sensitivity analysis by using 133 FDAapproved oncology drugs. C1, 2, and 5: CLL cells cultured alone as the control group. N1, 2, and 5: CLL cells cultured with NLCs. D The sensitivity of CLL cells from patients 1,2, and 5 to dactinomycin, carfilzomib, omacetaxine mepesuccinate, and venetoclax. The yellow box highlights the resistance to venetoclax of CLL cells co-cultured with NLCs.

by anti-Wnt5a antibody. These data provide novel mechanisms for venetoclax resistance and potential therapeutic options overcoming the resistance.

Previously, we reported that Wnt5a presents at high levels in patients with CLL compared with health donors [21, 40]. We speculate that the high-level Wnt5a noted in the plasma of patients with CLL is produced primarily by NLCs, which reside in lymphoid tissues. If so, then Wnt5a present in the plasma of patients with CLL may extend the protective effects of NLCs beyond the CLL microenvironment in which NLCs reside. Treatment targeting Wnt5a-signaling may mitigate such effects, potentially enhancing the clearance of leukemia cells when used 
A

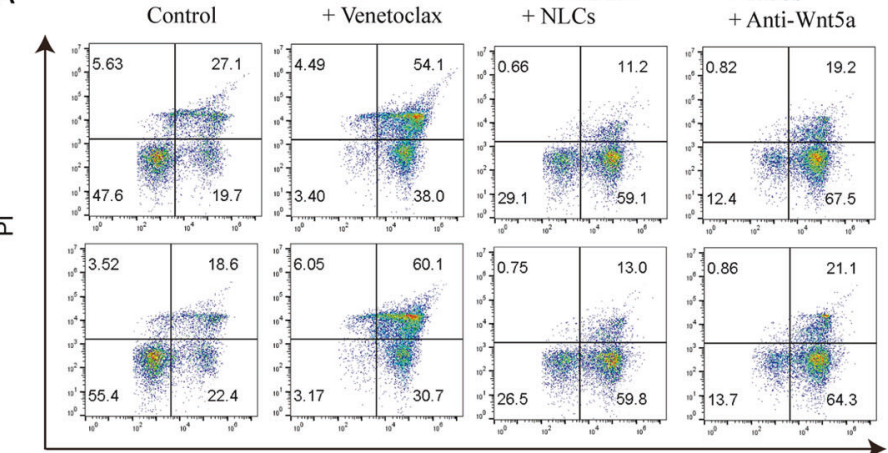

Annexin V
B

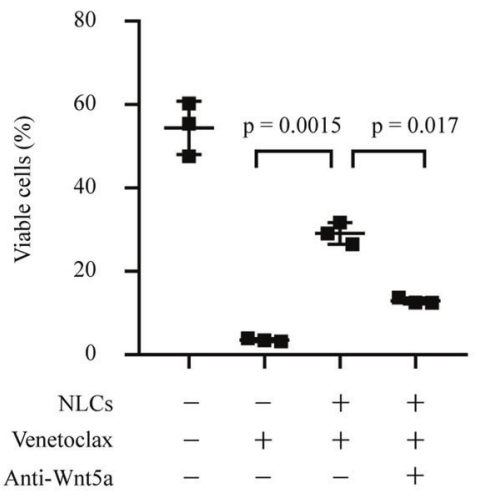

C

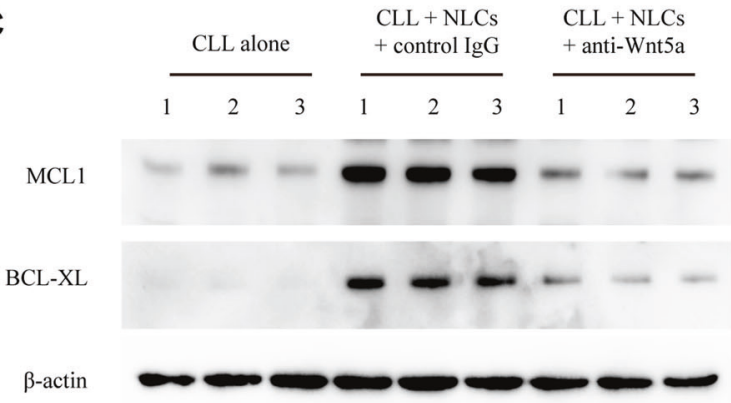

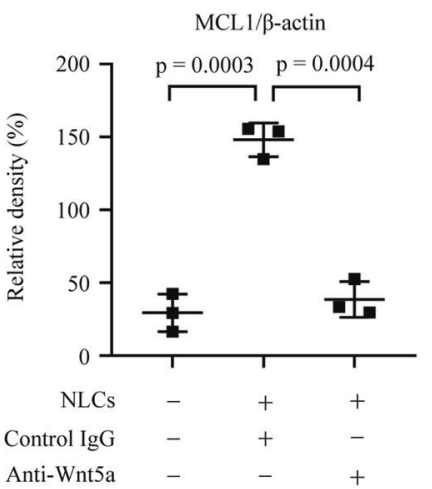

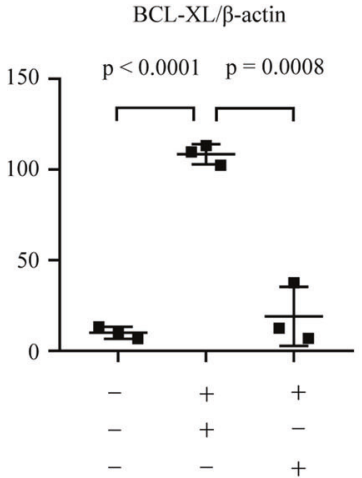

Fig. 5 NLCs upregulate MCL1 and BCL-XL in CLL cells through Wnt5a. A Flow cytometry analysis of apoptosis of CLL cells cultured with venetoclax, NLCs, and anti-Wnt5a for $24 \mathrm{~h}$. Dot figures represent viable cells in each group $(n=3)$. B Immunoblot analysis of MCL1 and BCL-XL in lysates of CLL cells with or without NLCs treated by anti-Wnt5a or control mAb of irrelevant specificity for $24 \mathrm{~h}$. Dot figures represent ratios of densities (MCL1/ $\beta$-actin and $\mathrm{BCL}-\mathrm{XL} / \beta$-actin).

alone or in combination with drugs that target other survivalsignaling pathways in CLL.

\section{METHODS}

\section{CLL specimens}

Blood samples were collected from The Seventh Affiliated Hospital, Sun Yat-sen University. Patients were provided written informed consent using a protocol approved by the Institutional Review Board of The Seventh Affiliated Hospital, Sun Yat-sen University, in accordance with the Declaration of Helsinki. Peripheral blood mononuclear cells (PBMCs) were isolated by density-gradient centrifugation with Ficoll-Paque PLUS (GE Healthcare Life Sciences, PA, USA), followed by purification with human B Cell Isolation Kit (130-091-151, Miltenyi Biotec Inc., CA, USA). Plasma was collected from blood samples that had undergone centrifugation for $10 \mathrm{~min}$ at $187 \times \mathrm{g}$ and stored at $-80^{\circ} \mathrm{C}$.

\section{Generation of nurse-like cells}

PBMCs isolated by Ficoll-Paque PLUS from CLL patients were suspended in RPMI with $20 \% \mathrm{FBS}$ to a final concentration of $2 \times 10^{7} / \mathrm{ml}$, as described [13]. After 14 days, the non-adherent CLL cells were removed by vigorously pipetting the contents of the well, leaving the adherent cells untouched. We observed these cells to have the morphology typical of NLC via microscopy.

\section{Materials}

133 FDA-approved oncology drugs were selected from FDA-approved Drug Library (L1300) of Selleckchem (TX, USA).

\section{Immunoblot analysis}

Immunoblot analysis was performed as described [41]. Primary mAb for $\beta$-actin (\#4970), ERK (\#4695), phospho-ERK (Thr202/Tyr204) (\#4370), HS1
(\#3892), phospho-HS1 (Tyr397) (\#8714), p65 (\#8242), phospho-p65 (Ser536) (\#3033), MCL-1 (\#5453), and BCL-XL (\#15071) were from Cell Signaling Technology (MA, USA). mAb (MAB645) for Wnt5a was from R\&D Systems (MN, USA). Secondary antibody conjugated with horseradish peroxidase was obtained from Cell Signaling Technology. The original western blots are provided (Supplemental Material, Supplementary Full Blots).

\section{Apoptotic analysis}

Apoptotic analysis was performed as described [42]. CLL cells were cultured with or without NLCs or anti-Wnt5a antibody (MAB645; R\&D Systems) $(5 \mu \mathrm{g} / \mathrm{mL})$ in RPMI culture-media, which were analyzed by Annexin V FITC Apoptosis Detection Kit I (BD Biosciences) and FACSCalibur Flow Cytometer (BD Biosciences). FlowJo (V10, FlowJo, OR, USA) was used for the analysis of apoptosis.

\section{Fluorescent microscopy}

Cells were stained with anti-CD163-PE (BD Biosciences, CA, USA) at $4{ }^{\circ} \mathrm{C}$ for $20 \mathrm{~min}$, washed twice with FACS buffer, and then fixed with $4 \%$ paraformaldehyde for $20 \mathrm{~min}$ at $4{ }^{\circ} \mathrm{C}$. Cells were washed twice with PBS and permeabilized with $0.3 \%$ Triton X-100 in PBS at $4^{\circ} \mathrm{C}$ for 10 min. After the cells were washed twice with PBS, they were blocked with $5 \% \mathrm{BSA}$ in PBS at $4^{\circ} \mathrm{C}$ for $30 \mathrm{~min}$. Anti-Wnt5a-FITC (Aviva Systems Biology, CA, USA) and anti-CD68-perCP (BioLegend, CA, USA) were added in blocking buffer and incubated overnight at $4{ }^{\circ} \mathrm{C}$. Cells were washed twice with PBS and incubated at room temperature for $60 \mathrm{~min}$. Cells were washed twice with PBS, and DAPI was added and incubated at room temperature for $10 \mathrm{~min}$. Images were collected on a Nikon A1R confocal microscope.

\section{Migration assay}

The migration assay across $5.0 \mu \mathrm{m}$ pore size polycarbonate membranes was performed as described [21]. A total of $5 \times 10^{5}$ cells were starved 

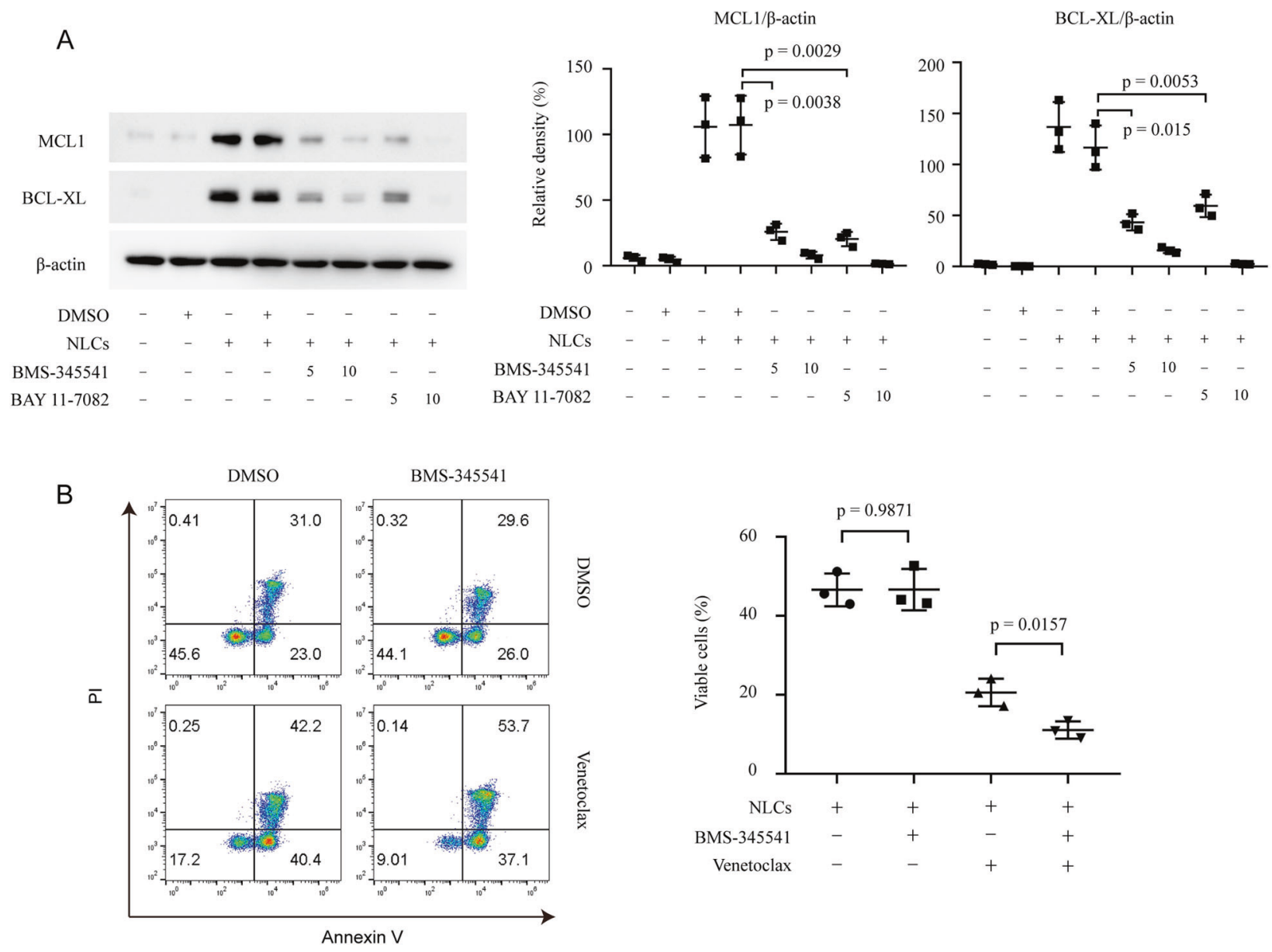

Fig. 6 NF-KB activation is required in NLCs-induced resistance to venetoclax in CLL cells. A Immunoblot analysis of MCL1 and BCL-XL in lysates of CLL cells with or without NLCs treated by BMS-345541 or BAY 11-7082. Bar figures represent ratios of densities (MCL1/ $\beta$-actin and $\mathrm{BCL}-\mathrm{XL} / \beta$-actin, $n=3$ ). B Flow cytometry analysis of apoptosis of CLL cells cultured with NLCs with $50 \mathrm{nM}$ venetoclax and $5 \mu \mathrm{M}$ BMS-345541. Dot figures represent the percentage of viable cells in each group $(n=3)$.

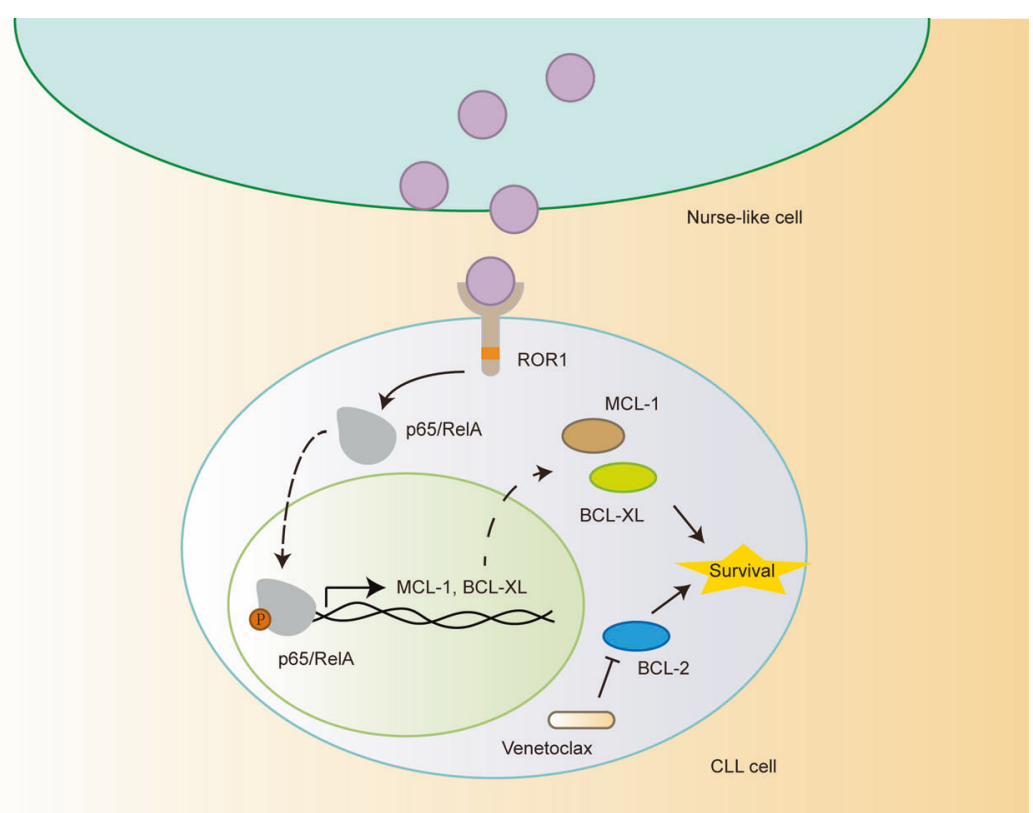

Fig. 7 The model for resistance to venetoclax in CLL cells. NLCs-Wnt5a-induced activation of NF- $\kappa B$ drives resistance to venetoclax in CLL cells through upregulation of MCL1 and BCL-XL. 
overnight and seeded in the upper compartment of transwell inserts (Corning, NY, USA). Cells were incubated for $4 \mathrm{~h}$ in serum-free medium with mAb neutralizing Wnt5a or control IgG at $37^{\circ} \mathrm{C}$ and $5 \% \mathrm{CO}_{2}$, and the migration toward NLCs was analyzed by microscopy. The percentage of migrating cells was calculated as the number of migrated cells in response to chemokine divided by the total number of input cells.

\section{ELISA}

Wnt5a ELISA kit (Aviva Systems Biology, CA, USA) was used to measure Wnt5a levels in plasma from patients with CLL per the manufacturer's instruction.

\section{High-throughput drug sensitivity analysis}

Primary CLL cells were cultured alone or with NLCs with RPMI with $10 \%$ FBS in 96-well plates. 133 oncology drugs were added into wells with final concentrations of $10 \mathrm{nM}, 100 \mathrm{nM}$, and $1000 \mathrm{nM}$. CLL cells cultured with drugs or DMSO $(<0.1 \%)$ as the vehicle were maintained in a humidified atmosphere at $37^{\circ} \mathrm{C}$ with $5 \% \mathrm{CO}_{2}$. Cells were stained with $0.5 \mu \mathrm{g} / \mathrm{ml}$ Propidium lodide before subjected to analysis. The Stratedigm S1400Exi flow cytometer platform with A600 plate loader was used for highthroughput flow cytometry analysis. FlowJo (V10, FlowJo, OR, USA) was used for the analysis of percentage of live cells and $R$ version 3.5.3 with pheatmap package (version 1.0.12) was used to generate heatmaps.

\section{Statistical analyses}

Data are presented as the mean \pm SD. Differences between two groups were determined by two-tailed Student's t-test. Correlation between two groups was determined by Pearson correlation coefficients. Significance was analyzed by GraphPad Prism 6.0 (GraphPad Software Inc., CA, USA) and $p$ values of less than 0.05 were considered significant.

\section{DATA AVAILABILITY}

All data generated or analyzed during this study are included in this published article and its supplementary information files.

\section{REFERENCES}

1. Kipps TJ, Stevenson FK, Wu CJ, Croce CM, Packham G, Wierda WG, et al. Chronic lymphocytic leukaemia. Nat Rev Dis Prim. 2017;3:16096.

2. Siegel RL, Miller KD, Jemal A. Cancer statistics, 2016. CA Cancer J Clin. 2016;66:7-30.

3. Liu $D$, Zhao J. Frontline therapies for untreated chronic lymphoid leukemia. Exp Hematol Oncol. 2019;8:15.

4. Hardy-Abeloos C, Pinotti R, Gabrilove J. Ibrutinib dose modifications in the management of CLL. J Hematol Oncol. 2020;13:66.

5. Perini GF, Ribeiro GN, Pinto Neto JV, Campos LT, Hamerschlak N. BCL-2 as therapeutic target for hematological malignancies. J Hematol Oncol. 2018;11:65.

6. Mihalyova J, Jelinek T, Growkova K, Hrdinka M, Simicek M, Hajek R. Venetoclax: A new wave in hematooncology. Exp Hematol. 2018;61:10-25.

7. Mato AR, Thompson M, Allan JN, Brander DM, Pagel JM, Ujjani CS, et al. Real-world outcomes and management strategies for venetoclax-treated chronic lymphocytic leukemia patients in the United States. Haematologica 2018;103:1511-7.

8. Bojarczuk K, Sasi BK, Gobessi S, Innocenti I, Pozzato G, Laurenti L, et al. BCR signaling inhibitors differ in their ability to overcome $\mathrm{Mcl}-1$-mediated resistance of CLL B cells to ABT-199. Blood. 2016;127:3192-201.

9. Elias $E E$, Almejun $M B$, Colado A, Cordini G, Vergara-Rubio M, Podaza E, et al. Autologous T-cell activation fosters ABT-199 resistance in chronic lymphocytic leukemia: rationale for a combined therapy with SYK inhibitors and anti-CD20 monoclonal antibodies. Haematologica. 2018;103:e458-e61.

10. Al-Sowayan BS, Al-Shareeda AT, Al-Hujaily EM. Exosomes, cancer's little army. Stem Cell Investig. 2019;6:9.

11. Kipps TJ, Choi MY. Targeted therapy in chronic lymphocytic leukemia. Cancer J. 2019;25:378-85.

12. Ferrer G, Montserrat E. Critical molecular pathways in CLL therapy. Mol Med. 2018:24:9.

13. Chen Y, Chen L, Yu J, Ghia EM, Choi MY, Zhang L, et al. Cirmtuzumab blocks Wnt5a/ROR1 stimulation of NF-kappaB to repress autocrine STAT3 activation in chronic lymphocytic leukemia. Blood. 2019;134:1084-94.

14. Burger JA, Tsukada N, Burger M, Zvaifler NJ, Dell'Aquila M, Kipps TJ. Blood-derived nurse-like cells protect chronic lymphocytic leukemia B cells from spontaneous apoptosis through stromal cell-derived factor-1. Blood. 2000;96:2655-63.
15. Tsukada N, Burger JA, Zvaifler NJ, Kipps TJ. Distinctive features of "nurselike" cells that differentiate in the context of chronic lymphocytic leukemia. Blood. 2002;99:1030-7.

16. Ferretti $E$, Bertolotto $M$, Deaglio $S$, Tripodo $C$, Ribatti $D$, Audrito $V$, et al. A novel role of the CX3CR1/CX3CL1 system in the cross-talk between chronic lymphocytic leukemia cells and tumor microenvironment. Leukemia. 2011:25:1268-77.

17. Sanchez-Lopez E, Ghia EM, Antonucci L, Sharma N, Rassenti LZ, Xu J, et al. NFkappaB-p62-NRF2 survival signaling is associated with high ROR1 expression in chronic lymphocytic leukemia. Cell Death Differ. 2020;27:2206-16.

18. Nishio M, Endo T, Tsukada N, Ohata J, Kitada S, Reed JC, et al. Nurselike cells express BAFF and APRIL, which can promote survival of chronic lymphocytic leukemia cells via a paracrine pathway distinct from that of SDF-1alpha. Blood. 2005;106:1012-20.

19. Fukuda $T$, Chen L, Endo T, Tang L, Lu D, Castro JE, et al. Antisera induced by infusions of autologous Ad-CD154-leukemia B cells identify ROR1 as an oncofetal antigen and receptor for Wnt5a. Proc Natl Acad Sci USA. 2008;105: 3047-52.

20. Choi MY, Widhopf GF 2nd, Ghia EM, Kidwell RL, Hasan MK, Yu J, et al. Phase I Trial: Cirmtuzumab inhibits ROR1 signaling and stemness signatures in patients with chronic lymphocytic leukemia. Cell Stem Cell. 2018;22:951-9. e3

21. Yu J, Chen L, Cui B, Widhopf GF 2nd, Shen Z, Wu R, et al. Wnt5a induces ROR1/ ROR2 heterooligomerization to enhance leukemia chemotaxis and proliferation. J Clin Invest. 2016;126:585-98.

22. Yu J, Chen L, Chen Y, Hasan MK, Ghia EM, Zhang L, et al. Wnt5a induces ROR1 to associate with 14-3-3zeta for enhanced chemotaxis and proliferation of chronic lymphocytic leukemia cells. Leukemia 2017;31:2608-14.

23. Hasan MK, Yu J, Chen L, Cui B, Widhopf li GF, Rassenti L, et al. Wnt5a induces ROR1 to complex with HS1 to enhance migration of chronic lymphocytic leukemia cells. Leukemia. 2017;31:2615-22.

24. Hasan MK, Rassenti LZ, Widhopf GF, Yu J, Kipps TJ. Wnt5a causes ROR1 to complex and activate cortactin to enhance migration of chronic lymphocytic leukemia cells. Leukemia. 2018;33:653-61.

25. Hasan MK, Yu J, Widhopf GF 2nd, Rassenti LZ, Chen L, Shen Z, et al. Wnt5a induces ROR1 to recruit DOCK2 to activate Rac1/2 in chronic lymphocytic leukemia. Blood. 2018;132:170-8.

26. Ghia EM, Rassenti LZ, Neuberg DS, Blanco A, Yousif F, Smith EN, et al. Activation of hedgehog signaling associates with early disease progression in chronic lymphocytic leukemia. Blood. 2019;133:2651-63.

27. Zhao Y, Zhang D, Guo Y, Lu B, Zhao ZJ, Xu X, et al. Tyrosine kinase ROR1 as a target for anti-cancer therapies. Front Oncol. 2021;11:680834.

28. Rozovski U, Harris DM, Li P, Liu Z, Jain P, Ferrajoli A, et al. STAT3-induced Wnt5a provides chronic lymphocytic leukemia cells with survival advantage. J Immunol. 2019;203:3078-85.

29. Janovska $P$, Poppova L, Plevova K, Plesingerova $H$, Behal $M$, Kaucka $M$, et al. Autocrine signaling by Wnt-5a deregulates chemotaxis of leukemic cells and predicts clinical outcome in chronic lymphocytic leukemia. Clin Cancer Res. 2016;22:459-69.

30. Hasan MK, Ghia EM, Rassenti LZ, Widhopf GF, Kipps TJ. Wnt5a enhances proliferation of chronic lymphocytic leukemia and ERK1/2 phosphorylation via a ROR1/DOCK2-dependent mechanism. Leukemia. 2020;35:1621-30.

31. Haselager M, Thijssen R, West C, Young L, Van Kampen R, Willmore E, et al. Regulation of $\mathrm{Bcl}-\mathrm{XL}$ by non-canonical NF-kappaB in the context of CD40-induced drug resistance in CLL. Cell Death Differ. 2021;28:1658-68.

32. Jayappa KD, Portell CA, Gordon VL, Capaldo BJ, Bekiranov S, Axelrod MJ, et al. Microenvironmental agonists generate de novo phenotypic resistance to combined ibrutinib plus venetoclax in CLL and MCL. Blood Adv. 2017;1:933-46.

33. Zhou Y, Kipps TJ, Zhang S. Wnt5a signaling in normal and cancer stem cells. Stem Cells Int. 2017;2017:5295286.

34. Asem MS, Buechler S, Wates RB, Miller DL, Stack MS. Wnt5a signaling in cancer. Cancers. 2016;8:79.

35. Hayakawa Y, Ariyama H, Stancikova J, Sakitani K, Asfaha S, Renz BW, et al. Mist1 expressing gastric stem cells maintain the normal and neoplastic gastric epithelium and are supported by a perivascular stem cell niche. Cancer Cell. 2015;28:800-14.

36. Bakker ER, Das AM, Helvensteijn W, Franken PF, Swagemakers S, van der Valk MA, et al. Wnt5a promotes human colon cancer cell migration and invasion but does not augment intestinal tumorigenesis in Apc1638N mice. Carcinogenesis 2013;34:2629-38.

37. Seiter K, Stiefel MF, Barrientos J, Shaikh A, Ahmed N, Baskind P, et al. Successful treatment of ibrutinib-associated central nervous system hemorrhage with platelet transfusion support. Stem Cell Investig. 2016;3:27.

38. Tariq S, Tariq S, Khan M, Azhar A, Baig M. Venetoclax in the treatment of chronic lymphocytic leukemia: Evidence, expectations, and future prospects. Cureus. 2020;12:e8908. 
39. Farooqui AA, Ashraf A, Farooq TB, Anjum A, Rehman SU, Akbar A, et al. Novel targeted therapies for chronic lymphocytic leukemia in elderly patients: A systematic review. Clin Lymphoma Myeloma Leuk. 2020;20:e414-e26.

40. Cui B, Ghia EM, Chen L, Rassenti LZ, DeBoever C, Widhopf GF 2nd, et al. High-leve ROR1 associates with accelerated disease progression in chronic lymphocytic leukemia. Blood. 2016;128:2931-40.

41. Chen Y, Guo Y, Zhao W, Tina Ho WT, Fu X, Zhao ZJ. Identification of an orally available compound with potent and broad FLT3 inhibition activity. Oncogene. 2016;35:2971-8.

42. Guo Y, Chen Y, Xu X, Fu X, Zhao ZJ. SU11652 inhibits tyrosine kinase activity of FLT3 and growth of MV-4-11 cells. J Hematol Oncol. 2012;5:72.

\section{ACKNOWLEDGEMENTS}

We thank Dr. Kipps (University of California, San Diego, CA) for generous supports and provision of reagents. The work was supported by Guangdong Provincial Key Laboratory of Digestive Cancer Research (2021B1212040006). We also thank Natural Science Foundation of Guangdong Province (2014A0303013398) to support the design of the study, National Natural Science Foundation of China (NSFC, Grant No. 82000150), Medical Research Programs of Guangdong Province (A2020442), and Shenzhen Science and Technology Innovation Commission (JCY20180307150614412, JCYJ20190814164601648 and JCYJ20190812093601675) to support sample collection and analysis, and Sanming Project of Medicine in Shenzhen (No. SZSM201911004) to support the manuscript preparation and publication.

\section{AUTHOR CONTRIBUTIONS}

$Y C, H P, B L$, and $Y G$ conceived the project. $Y G, H P, D Z$, and $Y Z$ performed the experiments. $\mathrm{FW}, \mathrm{HS}, \mathrm{JH}, \mathrm{CC}, \mathrm{XX}, \mathrm{SW}$, and $Y G$ collected clinical samples. $Y G, \mathrm{HP}, \mathrm{PL}, \mathrm{CY}$, $\mathrm{CZ}$, and $\mathrm{YP}$ analyzed data. YG, HP, BL, CC, XX, and YC wrote the manuscript.

\section{COMPETING INTERESTS}

The authors declare no competing interests.

\section{ADDITIONAL INFORMATION}

Supplementary information The online version contains supplementary materia available at https://doi.org/10.1038/s41420-022-00884-y.

Correspondence and requests for materials should be addressed to Chun Chen, Xiaojun Xu or Yun Chen.

Reprints and permission information is available at http://www.nature.com/ reprints

Publisher's note Springer Nature remains neutral with regard to jurisdictional claims in published maps and institutional affiliations.

(i) Open Access This article is licensed under a Creative Commons Attribution 4.0 International License, which permits use, sharing, adaptation, distribution and reproduction in any medium or format, as long as you give appropriate credit to the original author(s) and the source, provide a link to the Creative Commons license, and indicate if changes were made. The images or other third party material in this article are included in the article's Creative Commons license, unless indicated otherwise in a credit line to the material. If material is not included in the article's Creative Commons license and your intended use is not permitted by statutory regulation or exceeds the permitted use, you will need to obtain permission directly from the copyright holder. To view a copy of this license, visit http://creativecommons. org/licenses/by/4.0/.

(c) The Author(s) 2022 